\title{
INTRODUCTION TO ROUTING PROTOCOLS
}

Nikhil Kumar ${ }^{(1)}$, Shah Singh ${ }^{(2)}$, Jagdeep Singh ${ }^{(3)}$, Sahil Kumar ${ }^{(4)}$

${ }^{(1,3,4)}$ Lecturer, Shaheed Bhagat Singh State Technical Campus (SBSSTC), Ferozepur.

${ }^{2}$ Research Scholar (Pursuing M. tech), North West Institute of Engineering and Technology, V.P.O Dhudike, Moga.

\section{ABSTRACT}

In this era of communication network, we can access any website or contact any person with the help of internet or telephone anywhere in the world without knowing where the website is hosted and without knowing where the person is located. This all is done within a few seconds very efficiently. But the question comes out how it is done? And the answer is that, it is only done with the help of network routing. In this paper we review the IP routing and its protocols.

\section{Council for Innovative Research}

Peer Review Research Publishing System

Journal: INTERNATION JOURNAL OF COMPUTERS AND TECHNOLOGY

Vol. 13, No. 8

editorijctonline@gmail.com

www.ijctonline.com, www.cirworld.com 


\section{INTRODUCTION}

Once we create the internetwork by connecting our WANs and LANs to a router, we'll need to configure the logical network address such as IP address to all hosts on the internetwork so that they can communicate across the internetwork [1]. Router sends the data in the form of packets between the two end devices and this is done with the process known as IP routing. IP routing is a process of sending the packets from source to destination .It is also a process of selecting the best path from source to destination.

\section{Types of protocols}

There are two types of protocols used in routing:

1. Routed protocols.

2. Routing protocols.

\section{Routed Protocols}

These protocols are used to send data packets over a route decided by routing protocols. For e.g. IP, IPX, Apple Talk in Macintosh.

\section{Routing protocols}

These protocols are used to find out the best route among other possible routes to reach destination .For e.g. RIP, IGRP, EIGRP, OSPF.

\section{Routing types}

There are three types of routing.

1. Static routing

2. Default routing

3. Dynamic routing

\section{Static Routing}

In this type of routing we have to set the routes manually. This type of routing is suitable for small networks and all the burden or responsibility of performance of network is on the network administrator. It is time consuming as we have to set the routes manually and is not suitable for large networks. Static routing is applied on directly connected devices. It is more reliable and secure. In this routing highly sophisticated routers are not used.

Syntax that is used to add a static route to a routing table [2]

Router (config) \#ip route [destination network] [mask] [next-hop address or exit interface] [administrative_distance] [permanent]

This list describes each command in the string:

Ip route: This command is used to create the static route.

Destination network: This describes the network address that is placing in the routing table.

Mask: The subnet mask being used on the network.

Next-hop address: The address of the next hop router that will receive the packet and forward it to the remote network.

Exit interface: Used in place of next hop address and shows up a directly connected route.

Administrative distance: By default value of administrative distance in static routes is 1 .

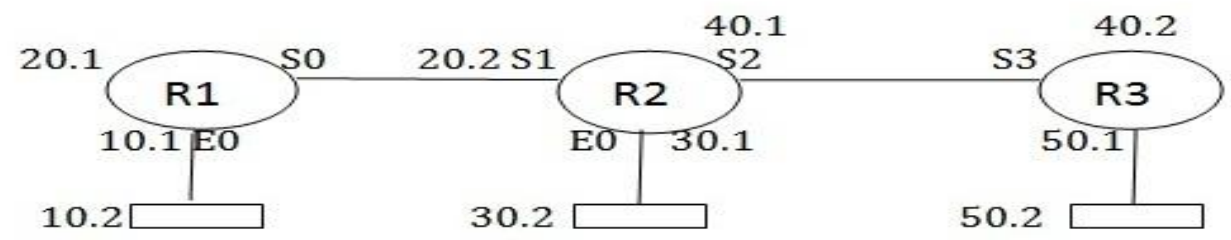

Fig.1

Router (config)\#ip route 30.0.0.0 255.0.0.0 S0 $1 \mathrm{P}$

If we set $P$ then while setting No ip route we have give full route again.

Router (config)\#ip route 30.0.0.0 255.0.0.0 20.0.0.2 $1 \mathrm{P}$ 
R1 (config)\#ip route 40.0.0.0 255.0.0.0 20.0.0.2

$\mathrm{R} 1$ (config)\#ip route 50.0.0.0 255.0.0.0 20.0.0.2

Administrative distance of static routing is 1 and both permanent and AD are optional

$\mathrm{R} 2$ (config)\#ip route 10.0.0.0 255.0.0.0 S1

$\mathrm{R} 2$ (config)\#ip route 10.0.0.0 255.0.0.0 20.0.0.1

$\mathrm{R} 2$ (config)\#ip route 50.0.0.0 255.0.0.0 40.0.0.2

R3(config)\#ip route 10.0.0.0 255.0.0.0 S3

R3(config)\#ip route 30.0.0.0 255.0.0.0 40.0.0.1

R3(config)\#ip route 20.0.0.0 255.0.0.0 40.0.0.1

\section{Default Routing}

This routing is performed only on the stub networks. Stub networks are those which has only one exit interface. Normally, if a specific route to a particular network does not exist, a router will drop all traffic destined to that network. A default route, or gateway of last resort, allows traffic to be forwarded, even without a specific route to a particular network [3].

The default route is identified by all zeros in both the network and subnet mask (0.0.0.0 0.0.0.0).It is the least specific route possible, and thus will only be used if a more specific route does not exist

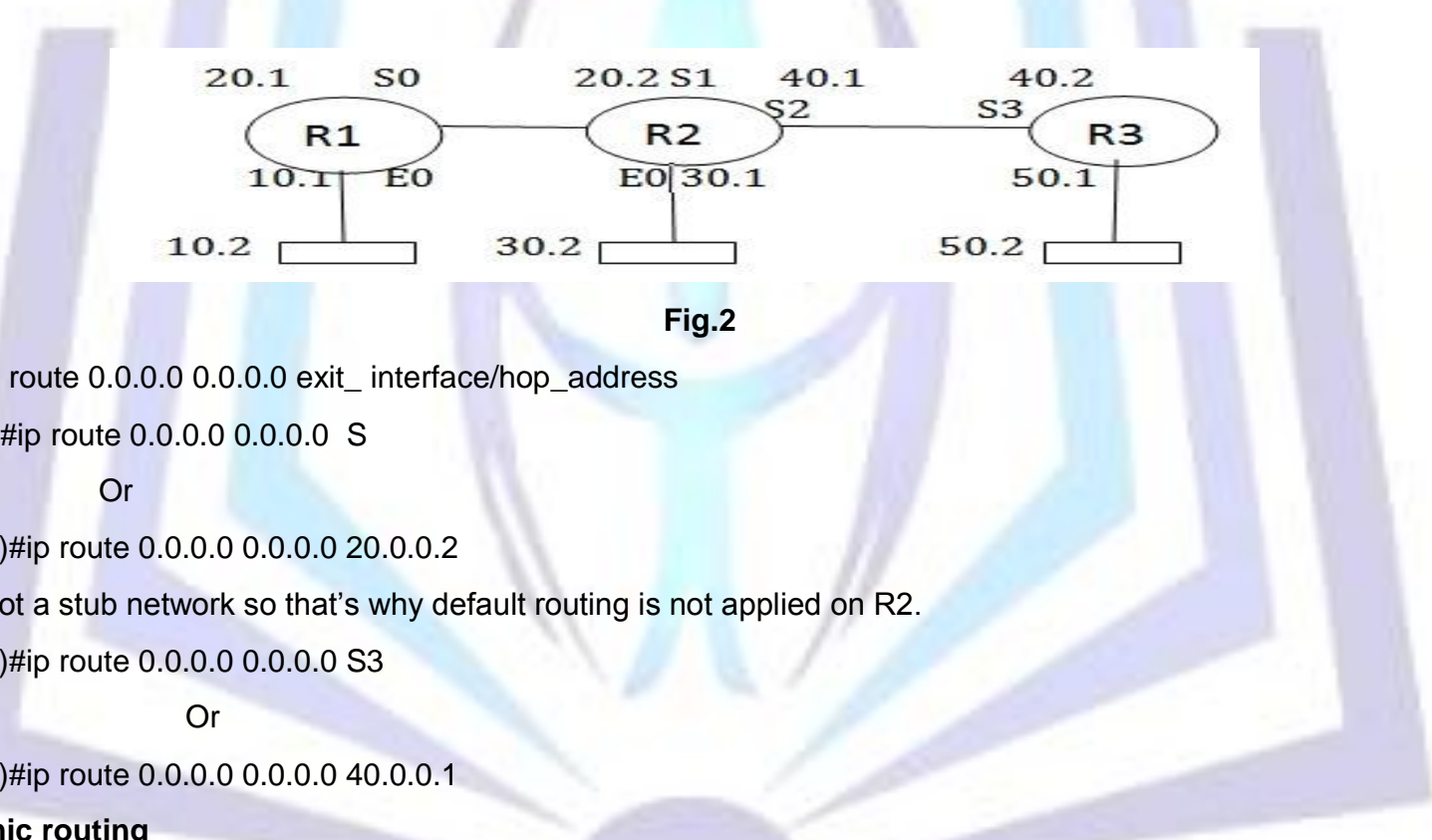

\section{Dynamic routing}

In case of dynamic routing a specific routing protocol is used and as a result router's routing table is configured and updated automatically. This type of routing is efficient for large networks but we need highly sophisticated routers and thus the cost of routing is very high. But there is also an advantage that the burden on administrator is less because everything is done automatically.

There are two categories of routing protocols:

1. IGP(Interior gateway protocol)

2. $\operatorname{EGP}($ Exterior gateway protocol)

1. IGP: In case of IGP all routers with same autonomous numbers (AS) will share the same routing table information or communicate with each other.

AS no.-It is a number that represents an area of which all the routers are members. It can be any number in the range of $1-65,535$.

e.g. -IGRP, EIGRP and OSPF.

2. EGP-It allows the routers with different autonomous number to communicate with each other. For e.g. BGP. 
3. Administrative distance (AD)-It is a metric that rates the trustworthiness and reliability of the routing information update being received either statically or dynamically. It can be any value in the range of $0-255$ where 0 is most reliable and 255 is unreliable.

$A D$ of directly connected network $=0$

$A D$ of static and default routing $=1$

$A D$ of $E I G R P=90$

$A D$ of IGRP $=100$

$A D$ of $O S P F=110$

$A D$ of $R I P=120$

There are three categories of routing protocols:

1. Distance vector routing protocols(DVRP)

2. Link state routing protocol(LSRP)

3. Hybrid routing protocol(HRP)

1.Distance vector routing- In this routing, each router passes its complete routing table information to its neighbours which then combine the received routing table entries with their own routing table to complete their own router's routing table. This information is just shared between those routers who are linked directly. This is information is shared at regular intervals. For e.g. after every 45 seconds each router sends its complete information to its neighbours. This routing is called routing by rumour because a router receiving an update from a neighbour router believes the information about remote networks without actually finding out for itself [4].

Some metrics are used to select the best path from source to destination to send the packet. And that metric is hop count. Lower the metric, the best the route is.

There are two types of distance vector routing protocols:

1. RIP

2. IGRP

RIP- Routing Information Protocol is a distance vector routing protocol. RIP sends whole routing table to its neighbours and it uses a hop count to determine the best way to a remote network.RIP has a maximum hop count of 16 and administrative distance is 120 . It is efficient in small networks. There are two versions of RIP.

RIP version 1- It is a class full routing which means all the devices in the network uses the same subnet mask. RIP version1 doesn't send updates with subnet mask information. It doesn't support VLSM.

RIP version 2- It is known as classless routing. It sends the updates along with subnet mask information. It is also called prefix routing. It supports VLSM.

To regulate the performance RIP uses timers which are listed as below:

1. Update timer- It is a timer after the expiry of which each router sends its complete routing table as an update to its immediate router. It is by default 30 secs.

2. Invalid timer- If no new update is received regarding a specific route entry the time period for which that route entry will be held waiting for a valid update is called invalid timer. It is by default 180 secs.

3. Hold down timer- If a route update is received regarding a specific route entry, that route entry has become unreachable then the time for which the route entry will be held waiting for a valid update that is called hold down timer. It is by default 180 secs.

4. Flush out timer- Once the route entry has become invalid the time period within which the router will intimate all its neighbours regarding the same is called flush out timer. It is by default 240 secs.

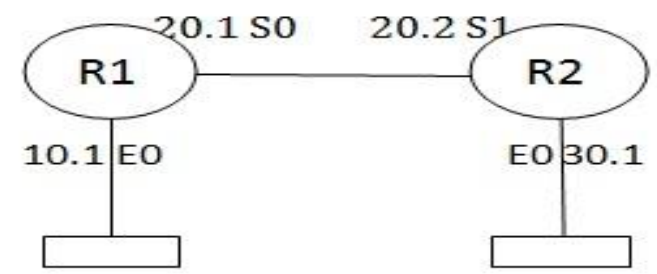

Fig.3 
Configuring RIP

R1(config)\#router rip

R1(config)\#network 10.0.0.0

R1(config)\#network 20.0.0.0

\section{R2(config)\#router rip}

R2(config)\#network 30.0.0.0

R2(config)\#network 20.0.0.0

2. IGRP-Interior Gateway Routing Protocol is a Cisco proprietary distance vector routing protocol which means to use this protocol all routers must be of Cisco [5]. It actually came into the power to remove the problems associated with RIP.IGRP has maximum hop count of 255 with default being 100. It is also efficient in large networks.IGRP uses bandwidth and delay as a metric to select the best way in determining the best route to a network. Its $A D=100$.It is a class full routing protocol. It doesn't support VLSM.

IGRP also has timers to regulate the performance which are same as that of RIP but with the difference of time period.

1. Update time: By default is 90 secs.

2. Invalid timer- 270 secs.

3. Hold down timer-280 secs.

4. Flush out timer-630 secs.

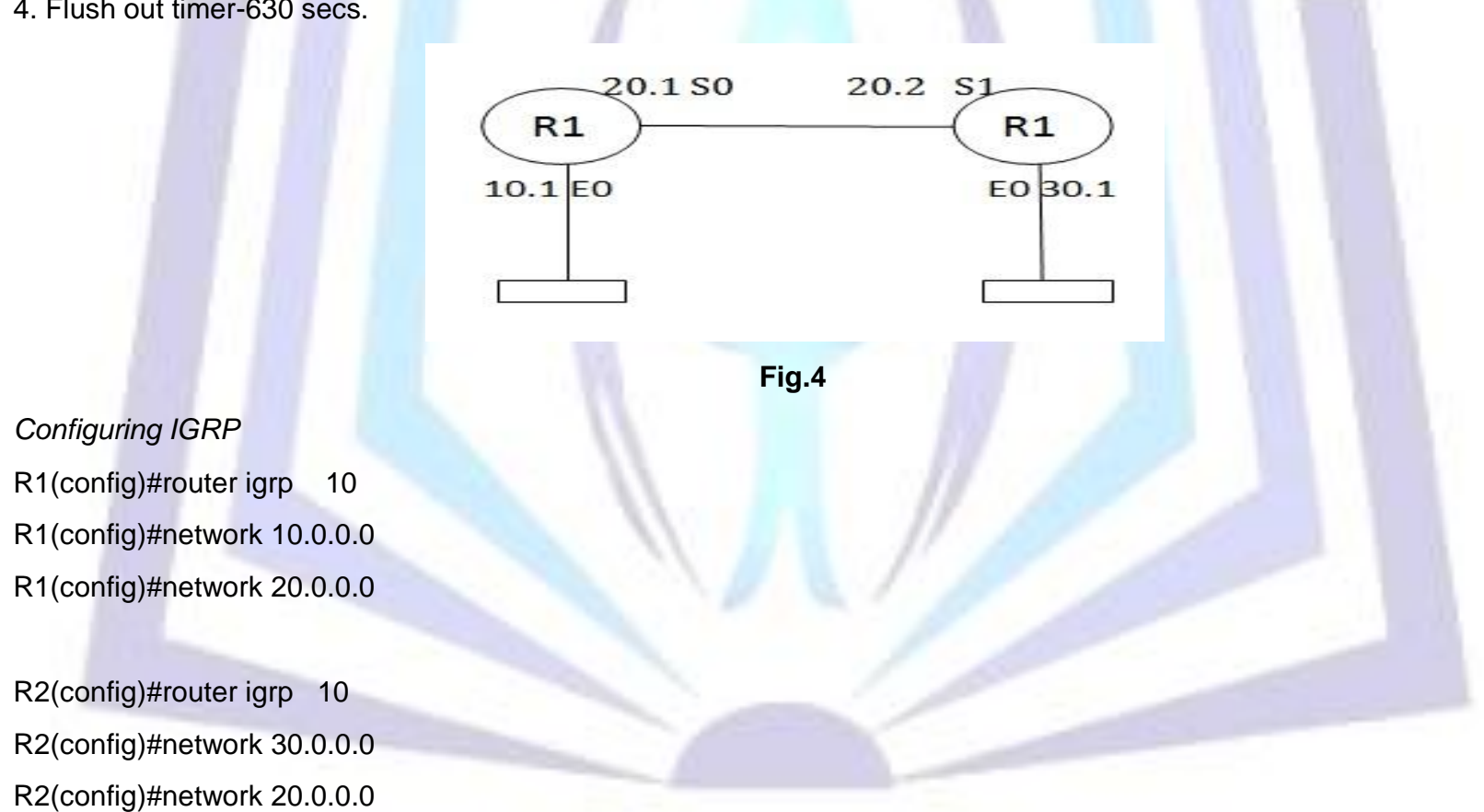

In above configuration of IGRP 10 is written and that is the autonomous number.

2. Link state routing protocol-Link state routing protocol is different from distance vector routing protocol. In link state routing, each router share its information of neighborhood with every other router on the network which means instead of sending complete routing table it just sends the information about its neighborhood only[6].Every router on the network receives a copy of same information multiple times. This is done by flooding. Flooding means that a router sends its neighbor information to all the routers on the network. Each neighbor sends the packet to its neighbor and so on. Every router that receives the packet sends the copies to its neighbors. In this way every router receives a copy of same information. For e.g. OSPF.

OSPF (Open shortest path first): It is a link state routing protocol. It's $A D=110$. It is an open standard routing protocol that provides multivendor support. It is a classless routing protocol. It supports VLSM.The metric used to identify the best route is bandwidth. It uses the concept of autonomous number and area. 
Wild card Mask (WCM): It is a difference between the full mask and subnet mask.

WCM=Full mask-Subnet mask

For e.g. WCM for 172.16.0.0

WCM=255.255.255.255-255.255.0.0

$=0.0 .255 .255$

For all IP addresses WCM $=0.0 .0 .0$

For e.g. WCM for 172.16.0.1=0.0.0.0

OSPF process ID: It is a locally significant; it represents a unique instance of OSPF over your router. It can be any number in between 1-65,535.

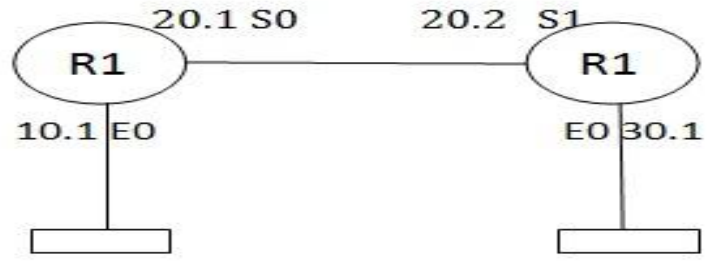

Fig.5

Configuring OSPF:

Router (config) \#router ospf OSPFprocessID

$\mathrm{R} 1$ (config) \#router ospf 10

R1 (config) \#network 10.0.0.0 0.255.255.255 area0

R1 (config) \#network 20.0.0.0 0.255.255.255 area0

R2 (config) \#router ospf 10

R2 (config) \#network 20.0.0.0 0.255.255.255 area0

R2 (config) \#network 30.0.0.0 0.255.255.255 area0

In the above configuration 10 is the OSPF process ID and area 0 is the main area or backbone area that manages the overall process of communication.

Some commands used in routing protocol are [7]:

1. Show ip ospf database-It is used to see the topology database table.

2. Show ip ospf neighbour-It is used to see the neighbour table.

3. Debugging: It is used to see the router backend processing at the frontend.

4. Debug ip RIP-It is used to see the backend processing relating to RIP at the frontend.

5. Debug ip IGRP event- It is used to see the backend processing relating to IGRP at the frontend

Which include request for update being made, sent and received?

6. Debug ip EIGRP-It is used to turn on the debugging relating to EIGRP.

3. Hybrid Routing Protocol: It combines the features of both DVRP and LSRP.for e.g. EIGRP

(a) EIGRP: Enhance Interior Gateway Routing Protocol is a hybrid routing protocol that combines the features of both DVRP and LSRP.It is a Cisco proprietary routing protocol .It is a classless routing protocol and supports VLSM.It uses the concept of AS no. Maximum hop count limit is 255 and AD is 90. 


\section{Characteristics of EIGRP:}

1. Protocol dependent modules- EIGRP supports multi network layer protocols like IP, IPX etc. Through PDM's.For each such protocol an independent set of database will be created.

2. Efficient Neighbour discovery- To become the immediate neighbour the hello packets should be exchanged, AS no's should be same, Metric should be identical. When the routers will become the immediate neighbours then only for the first time they will exchange their routing table with each other then only routing updates will be sent after regular intervals.

EIGRP three routing tables are prepared: Neighborship table, Topology table, Routing table. The metric used to identify the best path is a combination of four things:

1. Bandwidth-It should be more.

2. Delay-It should be less.

3. Load-It should be less.

4. Reliability-It should be more.

The best route to reach the destination network is called feasible distance.EIGRP supports multiple autonomous numbers.

\section{Configuring EIGRP:}

R1 (config)\#router EIGRP 10

R1(config)\#network 10.0.0.0

R1(config)\#network 20.0.0.0

R2(config)\#route EIGRP 10

R2(config)\#network 20.0.0.0

R2(config)\#network 30.0.0.0

Commands used on EIGRP protocol [8]:

Show IP route EIGRP: It is used to see EIGRP related entries in routing table.

Show IP EIGRP neighbor-It is used to see the neighborship table.

Show IP EIGRP topology-It is used to see the topology table.

\section{REFERENCES}

1. Router valley-Default routing http://www.routeralley.com/ra/docs/default routing.pdf].

2. Todd Lammle, "Syntax of static routing".

3. Todd Lammle, "IP routing".

4. Todd Lammle, "Routing protocols": Distance vector routing protocol.

5. Todd Lammle, "Routing protocols": Interior gateway routing protocol.

6. Tata McGraw - Hill-Data Communication and networking, "Link state routing protocol".

7. Todd Lammle, "EIGRP and OSPF": Commands used in OSPF.

8. Todd Lammle, "EIGRP and OSPF": Commands used in EIGRP. 